\title{
NS5A-P32 Deletion in Hepatitis C Genotype 1b Infection is the Most Refractory Treatment-Mediated Amino Acid Change Exhibiting Resistance to all NS5A Inhibitors
}

\author{
Hayato Hikita, MD, $\mathrm{PhD}^{1}$ Tetsuo Takehara, MD, $\mathrm{PhD}^{1}$ \\ ${ }^{1}$ Department of Gastroenterology and Hepatology, Graduate School \\ of Medicine, Osaka University, Suita, Osaka, Japan \\ Address for correspondence Tetsuo Takehara, MD, PhD, Department \\ of Gastroenterology and Hepatology, Graduate School of Medicine, \\ Osaka University, 2-2 Yamadaoka, Suita 565-0871, Osaka, Japan \\ Semin Liver Dis 2020;40:143-153. \\ (e-mail: takehara@gh.med.osaka-u.ac.jp).
}

\begin{abstract}
NS5A-P32 deletion (P32del) is a resistance-associated amino acid change that has recently gained popularity in direct-acting antiviral treatment for chronic hepatitis $C$. Although not yet detected in naive patients, it appears in 5 to $10 \%$ of hepatitis $C$ genotype $1 \mathrm{~b}$ patients who fail to respond to daclatasvir/asunaprevir and sofosbuvir/ledipasvir treatments. In contrast to signature resistance-associated substitutions, such as substitutions at the

Keywords

- chorionic hepatitis $C$ (CHC)

- resistance-associated substitutions (RAS)

- cross-resistance

- P32del

- mutation NS5A-L31 and NS5A-Y93 positions, it shows complete resistance to all NS5A inhibitors in replicon and cell culture. Studies of humanized liver mice suggest that P32del retains good replication fitness and requires two classes of antivirals, except NS5A inhibitors, to be suppressed effectively. Patients with the P32del virus do not respond to glecaprevir/ pibrentasvir but do respond to sofosbuvir/velpatasvir/voxilaprevir, presumably to sofosbuvir + glecaprevir/pibrentasvir, and at least partially to sofosbuvir/velpatasvir + ribavirin. Attention should be given to P32del in patients who experience failure with any NS5A inhibitor, especially those with genotype $1 \mathrm{~b}$ infection.
\end{abstract}

Antiviral treatments for chronic hepatitis $C$ patients have advanced remarkably over the past 5 years. In the past, antiviral treatments were based on interferon (IFN), which sometimes caused severe adverse events, including flu-like symptoms and thrombocytopenia, and their therapeutic effects were not satisfactory. Currently, approximately 95\% of patients can achieve virologic cure by combination treatment with direct-acting antiviral agents (DAAs) without any severe adverse events. ${ }^{1-8}$ Conversely, the genetic barrier of DAAs is lower than that of IFNs, and resistance-associated amino acid changes are a new issue for patients who experience virologic failure to DAA therapy. ${ }^{9-11}$

In particular, NS5A inhibitors, which are key drugs in DAA therapy, have extensive cross-resistance across NS5A inhibitors. First-generation NS5A inhibitors, such as daclatasvir and ledipasvir, are not effective for signature resistance-associated substitution, NS5A-Y93H $\pm \mathrm{L} 31 \mathrm{M} / \mathrm{I} / \mathrm{V} .^{12}$ Second-generation NS5A inhibitors, including pibrentasvir and velpatasvir, are more effective for these signature resistance-associated substitutions than first-generation inhibitors. ${ }^{12,13}$

published online December 13, 2019
DOI https://doi.org/ 10.1055/s-0039-3402001. ISSN 0272-8087.
NS5A P32 deletion (P32del), which has never been reported in DAA-naive patients, has been reported in patients with hepatitis $C$ virus (HCV) genotype $1 \mathrm{~b}$ who have failed DAA therapy, including daclatasvir-containing and sofosbuvir/ledipasvir treatments. HCV with NS5A P32del shows high-level resistance to all commercial NS5A inhibitors and is persistent for a long time in patients with virologic failure to DAA therapy. The treatment for HCV with NS5A P32del has not yet been established. Thus, in the present review, we summarize the data that have been reported for NS5A P32del HCV.

\section{NS5A P32del HCV was Selected in Patients Who Fail to Respond to NS5A Inhibitor- Containing Therapies}

Although NS5A P32del HCV was not found in in vitro experiments using HCV replicon treated with daclatasvir, ${ }^{14-17}$ it was discovered in clinical patients treated with daclatasvircontaining therapy (-Table 1). In a clinical trial of daclatasvir
Copyright @ 2020 by Thieme Medical Publishers, Inc., 333 Seventh Avenue, New York, NY 10001, USA. Tel: +1(212) 760-0888.
License terms

(c) 1 


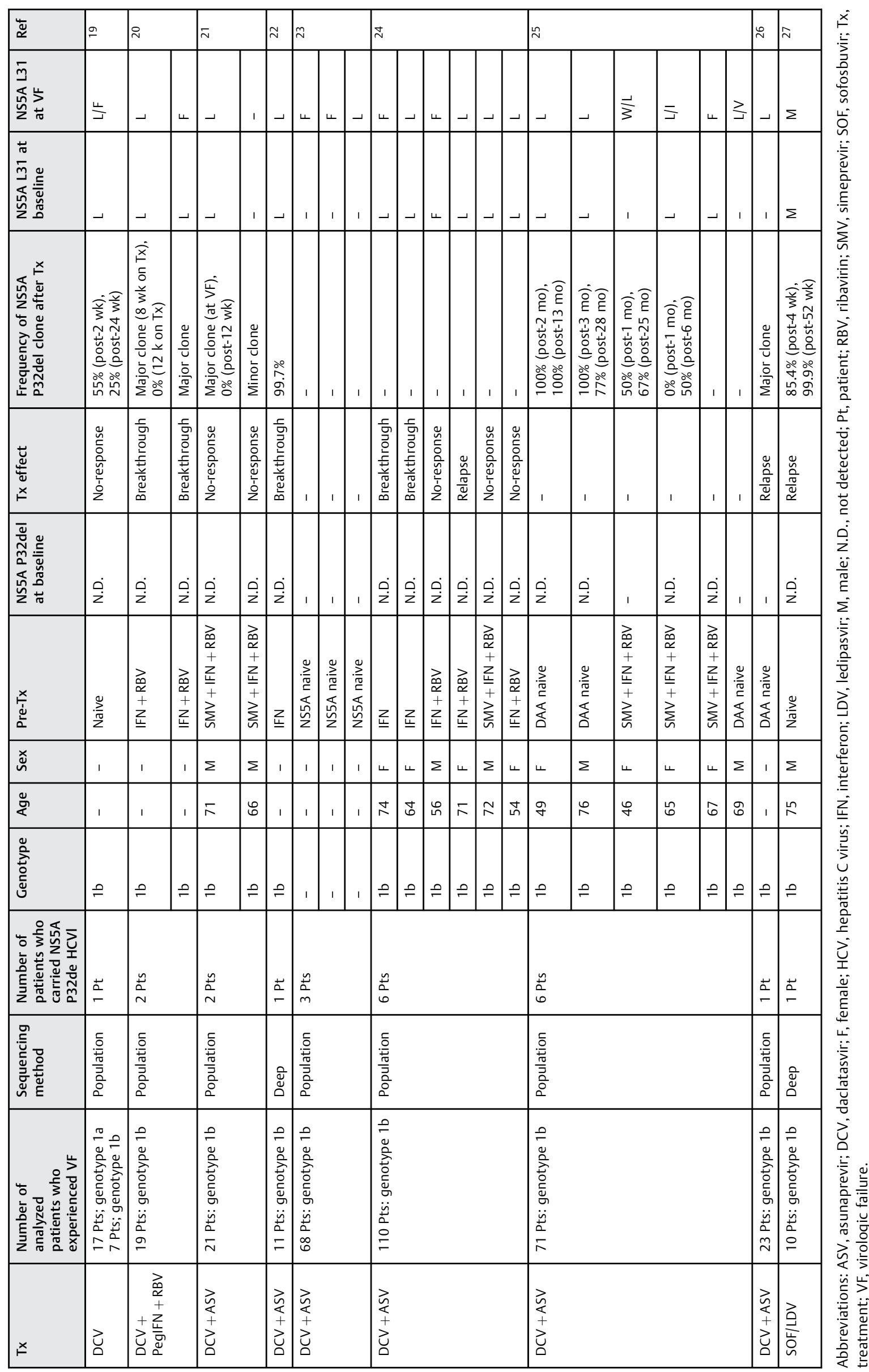


monotherapy, a total of 17 patients with genotype 1a HCV infection and 7 patients with genotype 1b HCV infection in the U.S. were treated with daclatasvir for 14 days. ${ }^{16,18}$ Various resistance-associated amino acid changes were detected after treatment. In one patient with genotype $1 \mathrm{~b} \mathrm{HCV}$ infection, NS5A P32del was detected as a major clone after treatment. ${ }^{19}$ In another clinical trial of daclatasvir + peg-IFN + ribavirin treatment in Japan, 19 prior IFN + ribavirin nonresponders with genotype $1 \mathrm{~b}$ HCV infection developed virologic failure. ${ }^{20}$ Among them, NS5A P32del was detected in two patients. One patient experienced viral breakthrough at 6 weeks after treatment but continued the treatment for 36 weeks. NS5A P32del was detected at 8 weeks after treatment, but it was no longer detected at 12 and 24 weeks after treatment or 4 weeks posttreatment. The other patient experienced viral breakthrough at 8 weeks after treatment but continued the treatment for 40 weeks. NS5A P32del was detected at 24 weeks after the initial treatment and at 4 weeks posttreatment. In both patients, NS5A P32del was not detected at baseline.

After the introduction of IFN-free DAA treatment for clinical use, Uchida et al reported that among 21 patients with genotype $1 \mathrm{~b} \mathrm{HCV}$ infection who experienced virologic failure by daclatasvir + asunaprevir, NS5A P32del was detected in two patients at the time of virologic failure. ${ }^{21}$ Both patients were prior simeprevir + IFN + ribavirin nonresponders. In one patient, HCV with NS5A P32del was detected as a minor clone and coexisted with a major clone of HCV carrying the NS5A P29 deletion at the time of virologic failure. In the other patient, it existed as a major clone at the time of virologic failure. In both patients, NS5A P32del was not detected by deep sequencing at baseline prior to daclatasvir + asunaprevir treatment or simeprevir + IFN + ribavirin treatment. Kai et al reported that among 11 patients with genotype $1 \mathrm{~b}$ HCV infection who experienced virologic failure by daclatasvir + asunaprevir, NS5A P32del was detected in one patient as a major clone at the time of virologic failure analyzed by deep sequencing. ${ }^{22}$ The patient had never received any DAAs, including simeprevir, prior to daclatasvir + asunaprevir treatment. By population sequencing analysis, Itakura et al, ${ }^{23}$ Kobayashi et al, ${ }^{24}$ and Iio et $\mathrm{al}^{25}$ reported that among 68, 110 , and 74 patients with genotype 1b HCV infection, respectively, who experienced virologic failure to daclatasvir + asunaprevir treatment, NS5A P32del was detected in 3, 6, and 7 patients at the time of virologic failure, respectively. Teraoka et al also reported that among 23 patients with genotype $1 \mathrm{~b}$ HCV infection who experienced virologic failure to daclatasvir + asunaprevir \pm beclabuvir treatment, NS5A P32del was detected in one patient treated with daclatasvir + asunaprevir at the time of virologic failure. ${ }^{26}$ These reports indicated that NS5A P32del was detected in 5 to $10 \%$ of patients with genotype $1 \mathrm{~b}$ $\mathrm{HCV}$ infection who failed to daclatasvir-containing therapies (-Table 1).

NS5A P32del was also reported after sofosbuvir/ledipasvir treatment. ${ }^{27}$ Doi et al reported that among 10 patients with genotype $1 \mathrm{~b} \mathrm{HCV}$ infection who experienced virologic failure to sofosbuvir/ledipasvir treatment, NS5A P32del was detected as a major clone in one patient at the time of virologic failure. ${ }^{27}$
Phylogenetic tree analysis clarified that the NS5A P32del HCV clones in this case were generated by the complete deletion of three bases, which encode NS5A P32 from the original HCV clones. The patient had not received any anti-HCV therapy and had liver cirrhosis with a history of hepatocellular carcinoma treatment. Conversely, there has not been a reported case of naturally occurring NS5A P32del without DAA treatment or a case infected with NS5A P32del HCV that was not genotype $1 \mathrm{~b}$ until now.

\section{NS5A P32del HCV Shows High-Level Resistance to all NS5A Inhibitors}

NS5A protein, an active component of HCV replicase, consists of domains I, II, and III, and domain I consists of the N-terminal membrane-anchoring helix, domain Ia and domain Ib (- Fig. 1). ${ }^{28-30}$ Domain I forms dimers, and domain Ib binds ribonucleic acid (RNA) for HCV replication. ${ }^{31}$ NS5A inhibitors bind NS5A domain I, leading to inhibition of RNA binding. ${ }^{31-33}$ Recently, NS5A domain I was reported to play a role not only in viral replication but also in viral assembly. ${ }^{34}$ P32 is located at the linker between the membrane-anchoring helix and domain Ia (-Fig. 1). NS5A inhibitors bind a space formed by the linker, domain I and the helix (- Fig. 1). ${ }^{32,33}$

Using the genotype $1 \mathrm{~b} \mathrm{HCV}$ replicon system (Con 1 replicon), NS5A P32del HCV showed extremely high-level resistance against daclatasvir and ledipasvir (-Table 2). ${ }^{13,19}$ The mean $\mathrm{EC}_{50}$ of daclatasvir or ledipasvir against NS5A P32del HCV is approximately 0.3 to 0.4 million times higher than that against wild-type HCV. ${ }^{13,19}$ In regard to NS5A L31M/V-Y93H double mutation $\mathrm{HCV}$, which is most frequently selected in patients who failed to respond to daclatasvir + asunaprevir $^{21-23,26}$ or sofosbuvir/ledipasvir, ${ }^{27}$ the mean $\mathrm{EC}_{50}$ of daclatasvir or ledipasvir against the double mutant $\mathrm{HCV}$ is approximately 7,000 to 75,000 times higher than that against wild-type $\mathrm{HCV}^{15-17,35}$ (-Table 2). NS5A P32del HCV shows higher-level resistance to daclatasvir or ledipasvir than the NS5A L31M/V-Y93H double mutation HCV. While HCV with the NS5A L31M/V-Y93H double mutation $\mathrm{HCV}$ is sensitive to a second-generation NS5A inhibitor, such as pibrentasvir ${ }^{13}$ and velpatasvir, ${ }^{36}$ NS5A P32del HCV shows high-level resistance against all NS5A inhibitors used in clinical practice, including ombitasvir, elbasvir, pibrentasvir, and velpatasvir. ${ }^{13}$ The mean $\mathrm{EC}_{50} \mathrm{~S}$ of second-generation NS5A inhibitors against NS5A P32del HCV are approximately 1,000 to 1.7 million times higher than those against wild-type HCV ( - Table 2 ). ${ }^{13}$

The JFH-1 HCV clone, genotype $2 \mathrm{a} \mathrm{HCV}$, can infect hepatoma cells and is useful as an in vitro infection model. Using JFH-1based recombinant HCV with NS5A from genotype1b HCV, HCV with NS5A P32del demonstrated high-level resistance to daclatasvir or ledipasvir, which is consistent with replicon studies (-Table 2). ${ }^{12,37,38}$ Importantly, while the NS5A $\mathrm{L} 31 \mathrm{M} / \mathrm{V}-\mathrm{Y} 93 \mathrm{H}$ double mutation $\mathrm{HCV}$ is relatively sensitive to second-generation NS5A inhibitors, including velpatasvir and pibrentasvir, NS5A P32del HCV still remains highly resistant to all second-generation NS5A inhibitors. ${ }^{12,37-39}$ The EC $_{50}$ of velpatasvir against NS5A P32del HCV is 1,000 to 40,000 times higher than that against wild-type HCV, while that 


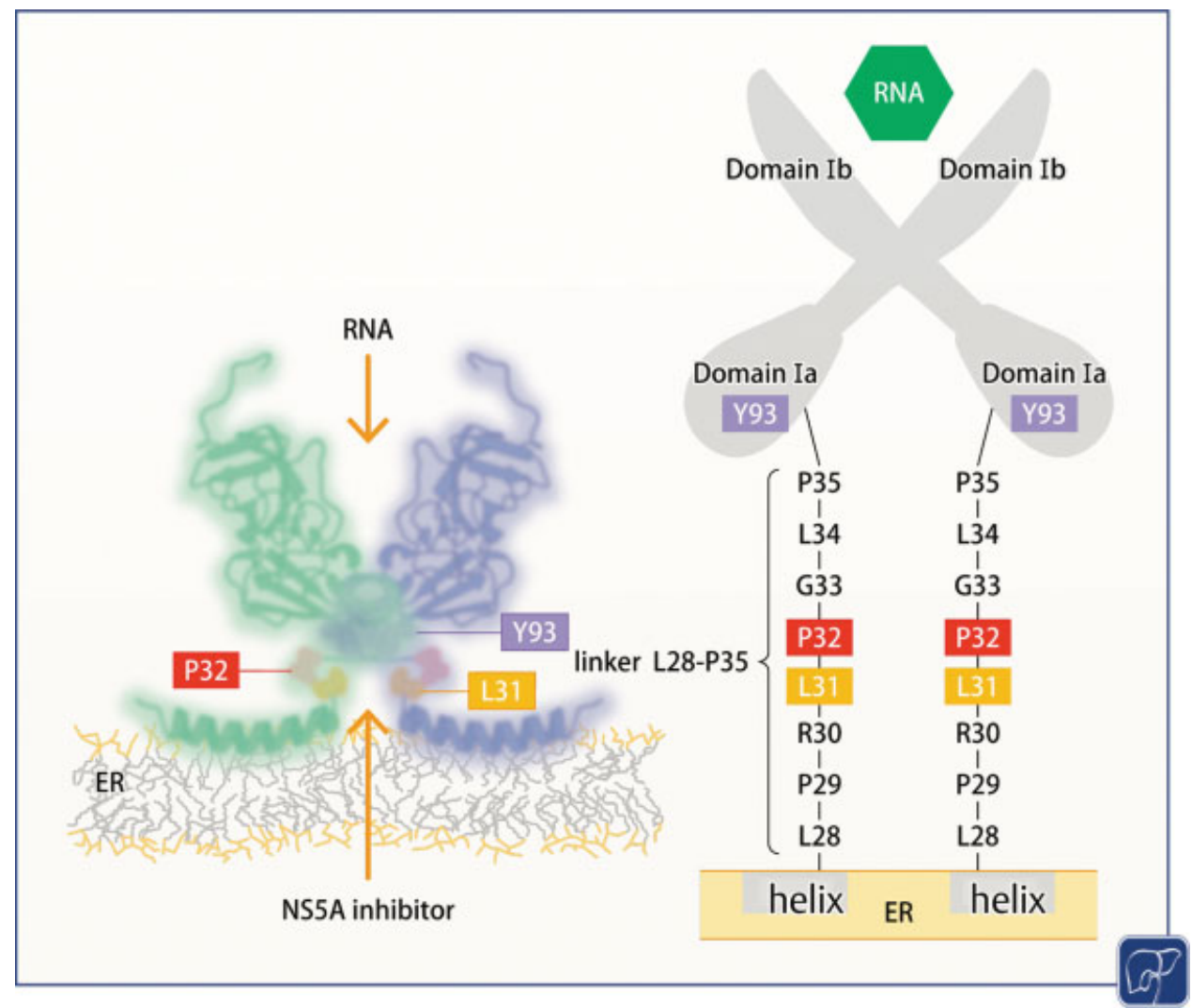

Fig. 1 Structure of NS5A domain I.

against NS5A L31M-Y93H HCV is 44 to 999 times higher than that against wild-type $\mathrm{HCV}$ (- Table 2). ${ }^{12,13}$ The $\mathrm{EC}_{50}$ of pibrentasvir against NS5A P32del HCV is 1,000 times higher than that against wild-type HCV, while that against NS5A L31M-Y93H HCV is 0.7 to 79 times higher than that against wild-type HCV (- Table 2). ${ }^{12,13}$ These data suggest that HCV with NS5A P32del, which is selected in patients with a failed response to an NA5A inhibitor, acquires cross-resistance to all other NS5A inhibitors. Conversely, the $\mathrm{EC}_{50}$ 's of other class antivirals, including NS3/4A inhibitors, NS5B inhibitors, ribavirin, and IFN, against NS5A P32del HCV are similar to those of wild-type HCV. ${ }^{37,38}$

\section{NS5A P32del HCV Replicates at a Low Level In Vitro but Persists in Patients}

In contrast to the extremely high-level resistance of NS5A P32del HCV to all NS5A inhibitors, its replication levels decrease to $30 \%$ when compared with replication levels of wildtype HCV in replicon systems. ${ }^{19}$ Similarly, the replication levels of NS5A P32del HCV are lower than those of wild-type HCV in the JFH-1-based recombinant $\mathrm{HCV}$ infection system (-Table 2). ${ }^{37,38}$ Despite the low replication data of NS5A P32del HCV in vitro, clinical data have shown that selected NS5A P32del HCV by DAA treatment failure remains in patients for more than 1 year. ${ }^{25,27}$ Amino acid substitutions of NS5A L31F and L31M are sometimes codetected in patients with NS5A P32del HCV (-Table 1). The results of in vitro experiments indicated that these substitutions increased the replication capacity of P32del HCV only slightly; however, the replication levels of N5A L31F/M + P32del HCV were still lower than those of wild-type HCV ( - Table 2). ${ }^{37,38}$ Doi et al reported that no other compensatory amino acid change was detected in NS5A domain 1 lesions in patients with P32del HCV. ${ }^{27}$ We cannot exclude the possibility that some other amino acid changes outside of the NS5A domain 1 contribute to replication fitness.

Examining HCV infection in vivo is difficult because HCV does not typically infect experimental small animals. However, mice whose hepatocytes are replaced by human hepatocytes have been used to establish an experimental model with several immune-deficient mouse strains. ${ }^{40-43}$ After sera from $\mathrm{HCV}$-infected patients or a chimpanzee are inoculated into them, the mice develop persistent viremia of HCV. ${ }^{43-47}$ Doi et al reported that after patient serum containing over 99\% of NS5A P32del HCV was inoculated into 25 humanized liver mice, all of them developed persistent viremia, with over 99\% carrying NS5A P32del HCV; no compensatory amino acid change was detected in NS5A domain 1 lesions. ${ }^{37}$ Their serum HCV RNA levels were similar to those of mice inoculated with patient serum containing wild-type HCV. ${ }^{37}$ Osawa et al also reported that after patient serum containing NS5A P32del HCV as the major clone was inoculated into humanized liver mice, they developed persistent viremia carrying NS5A P32del HCV as the major clone. ${ }^{48}$ These findings support the idea that the NS5A P32del that occurs selectively in patients who fail to respond to DAAs already obtained replication fitness. 


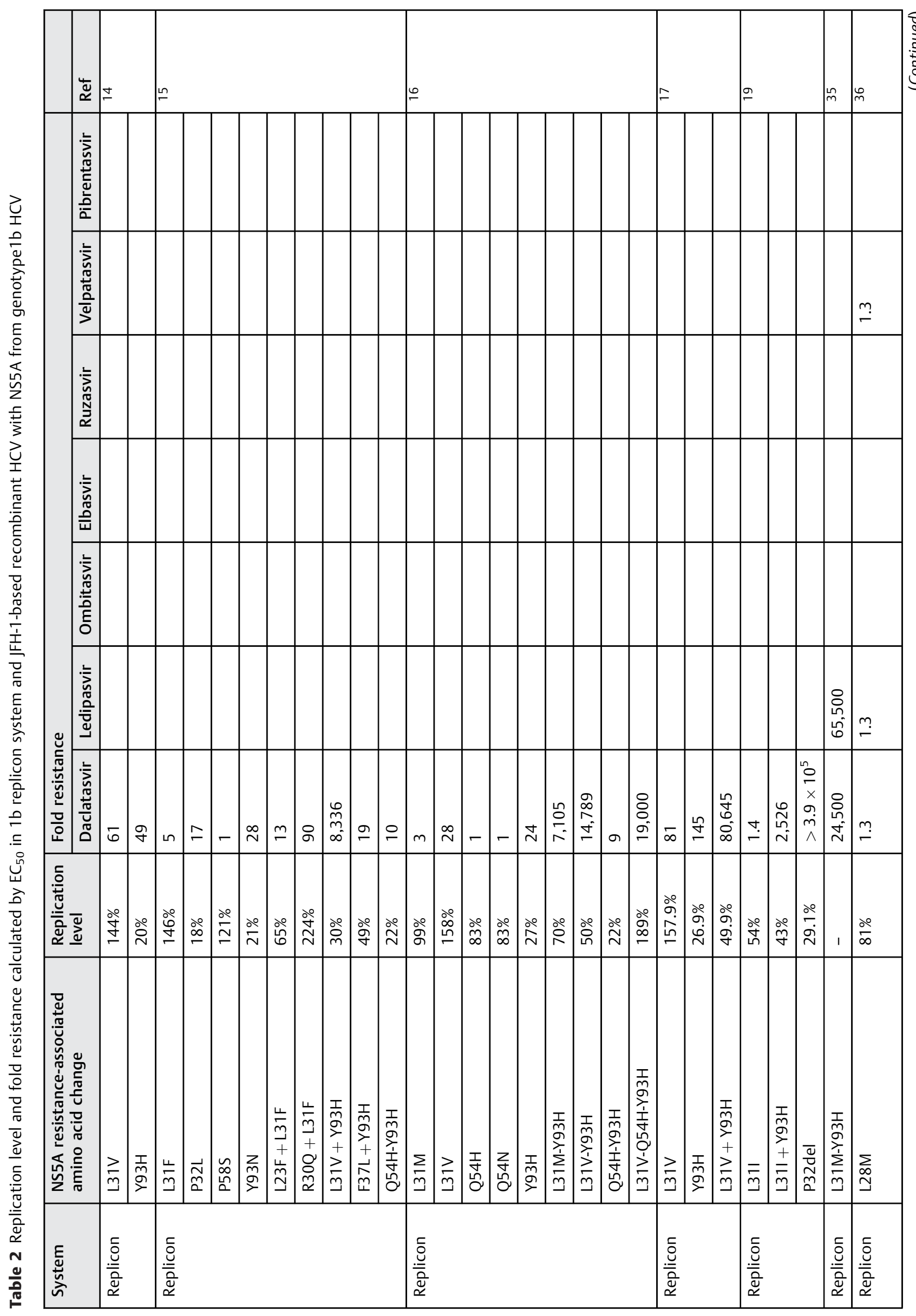




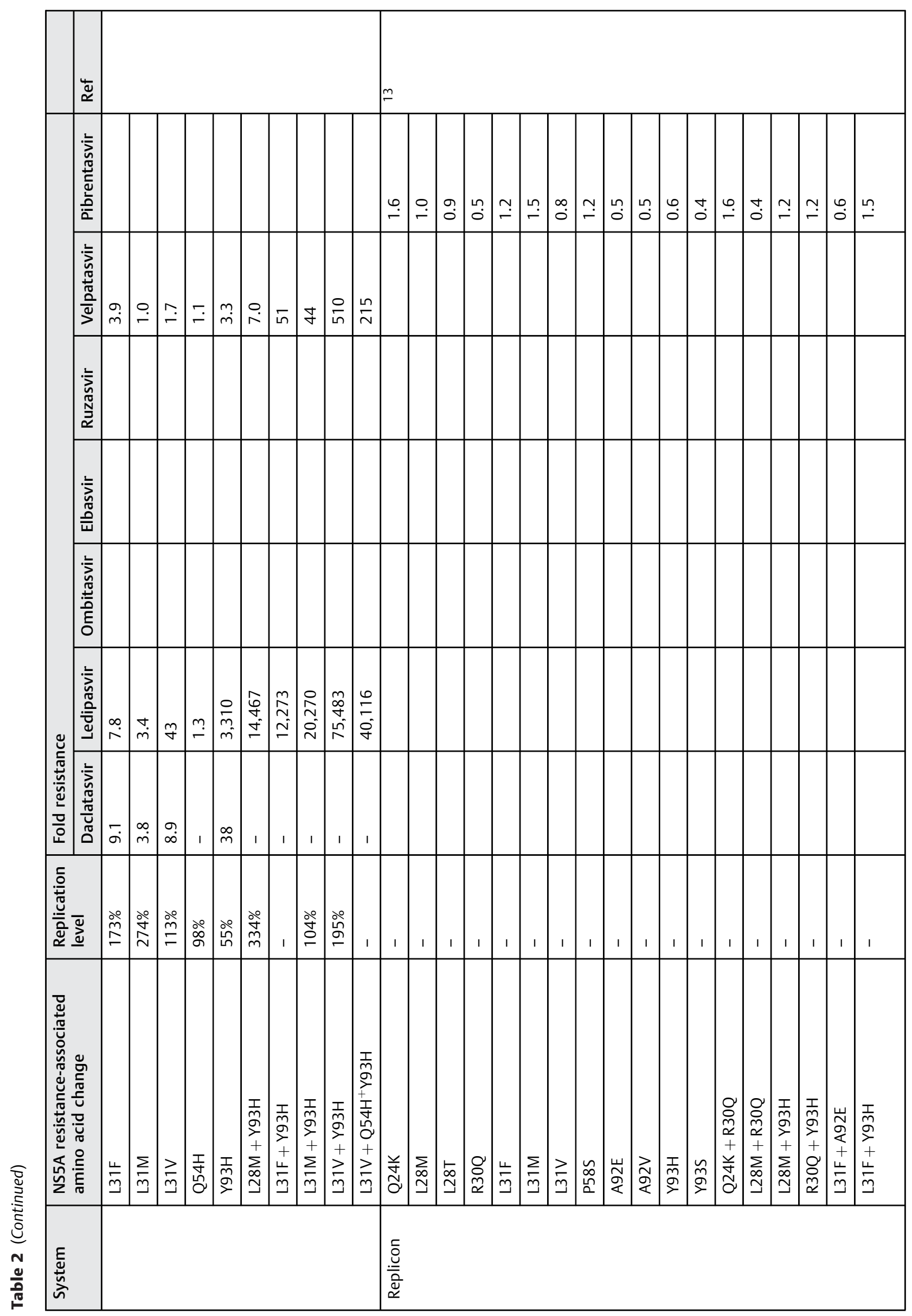




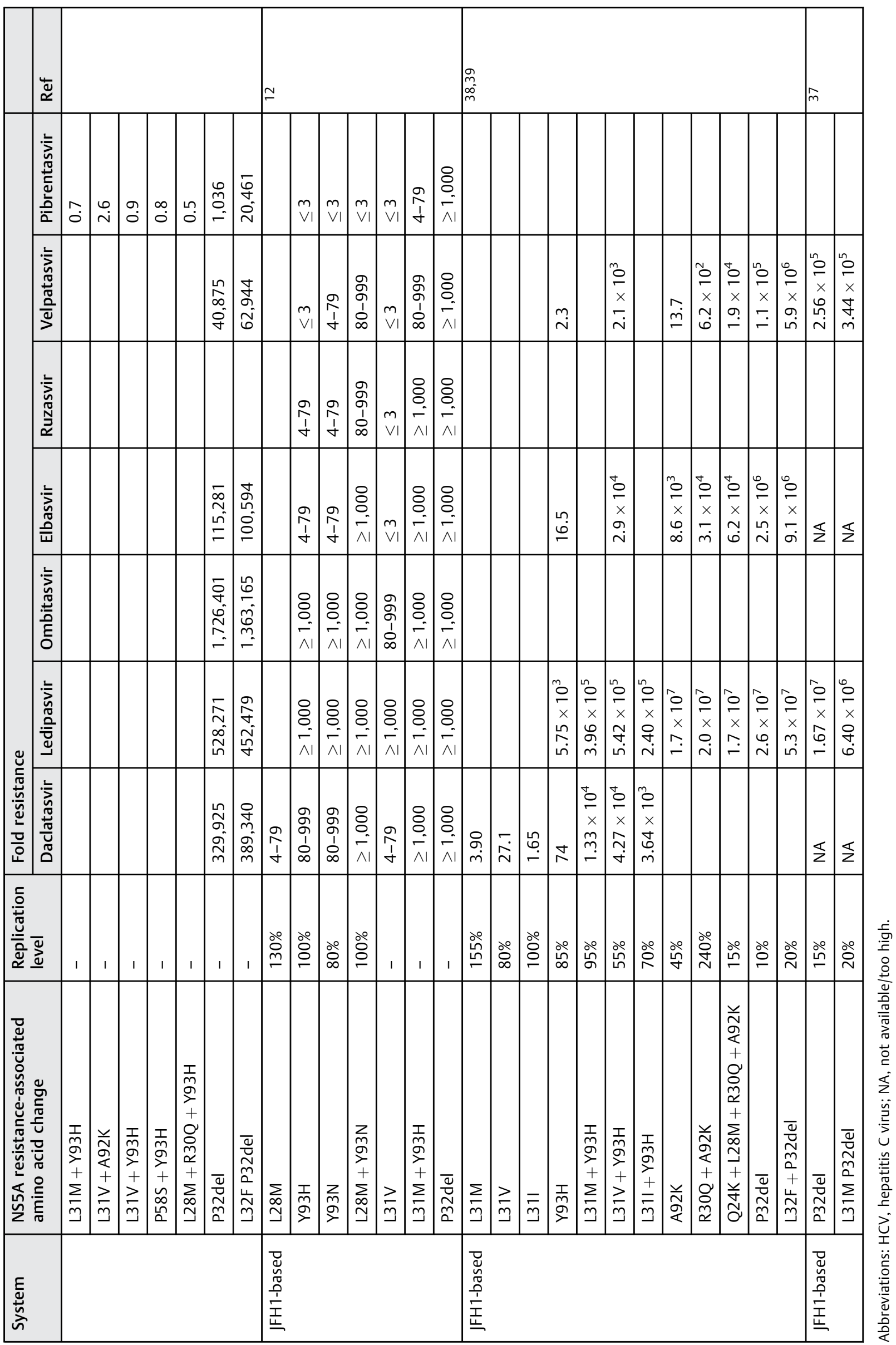




\section{Identifying Treatments that can Eliminate HCV with NS5A P32del}

The effects of some treatments against NS5A P32del HCV were examined using NS5A P32del HCV-infected humanized liver chimeric mice. Osawa et al reported that serum HCV RNA levels of NS5A P32del HCV-infected mice did not decrease to below the lower limit of detection levels with glecaprevir/pibrentasvir treatment, while both wild-type HCV-infected mice and L31M/ Y93H HCV-infected mice achieved sustained virologic response (SVR) with glecaprevir/pibrentasvir. ${ }^{48}$ Teraoka et al reported that NS5A P32del HCV-infected mice showed high-level resistance to glecaprevir/pibrentasvir treatment but were sensitive to the combination treatment of sofosbuvir + glecaprevir/ pibrentasvir. $^{26}$ Doi et al reported that NS5A P32del HCVinfected mice showed high-level resistance against the combination treatment ledipasvir + GS-558093, a nucleotide NS5B inhibitor, as well as ledipasvir monotreatment or elbasvir monotreatment. ${ }^{37}$ However, they showed sensitivity to the combination treatment simeprevir + GS-558093 or simeprevir + PegIFN. ${ }^{37}$ These data suggest that NS5A P32del HCV are difficult to eliminate with a combination treatment of two DAAs including an NS5A inhibitor such as sofosbuvir/ledipasvir or glecaprevir/pibrentasvir. Combination treatments containing more than one drug, except NS5A inhibitors, should be required for NS5A P32del HCV.

\section{Clinical Data on Retreatment with Sofosbuvir/Ledipasvir or Glecaprevir/Pibrentasvir in Patients with NS5A P32del HCV}

To date, some patients with NS5A P32del HCV have received retreatment with IFN-free DAA therapy (-Fig. 2). Akuta et al reported that a total of three patients were retreated by sofosbuvir/ledipasvir, and all three developed virologic failure. $^{49}$ Conversely, one patient with NS5A P32del HCV who achieved SVR with sofosbuvir/ledipasvir was reported. ${ }^{50}$ In addition, before retreatment, the patient had HCV that carried L31M, Q54H, A92K, and Y93H. In a phase 3 clinical trial of glecaprevir/pibrentasvir for HCV-infected Japanese patients with prior DAA experience (CERTAIN-1), a total of 32 patients were enrolled. ${ }^{51}$ Among them, two genotype $1 \mathrm{~b}$ HCV-infected patients who had experienced virologic failure to daclatasvir + asunaprevir had NS5A P32del HCV before retreatment with glecaprevir/pibrentasvir. Both patients developed virologic failure again by retreatment, while the other 30 patients achieved SVR. ${ }^{13,51,52}$ In a phase 3 clinical trial of glecaprevir/pibrentasvir in Europe (MAGELLAN-1), a patient infected with genotype $1 \mathrm{~b}$ HCV experienced virologic failure to daclatasvir + asunaprevir had NS5A P32del HCV before retreatment with glecaprevir/pibrentasvir and again developed virologic failure. ${ }^{53}$ For real-world data, Osawa et al reported cases in which 30 patients with a previous history of DAA therapy, including 1 patient with NS5A P32del HCV, were retreated with glecaprevir/pibrentasvir. ${ }^{54}$ A total of two patients, including the patient with NS5A P32del HCV, developed virologic failure. Uemura et al reported cases in which 42 patients with a previous DAA therapy history, including 3 patients with NS5A P32del HCV, were retreated with glecaprevir/pibrentasvir. ${ }^{55}$ All three patients with NS5A P32del HCV experienced virologic failure, while the other patients achieved SVR. Collectively, NS5A P32del HCV is highly resistant to glecaprevir/pibrentasvir as well as sofosbuvir/ledipasvir, which is consistent with the experimental results from the murine model.

\section{Clinical Data on Retreatments with Three DAAs and Sofosbuvir/NS5A Inhibitor Plus Ribavirin in Patients with NS5A P32del HCV}

Among treatments containing more than one drug but not NS5A inhibitors, the efficiencies of 4 types of treatments for patents with NS5A P32del HCV have been reported:

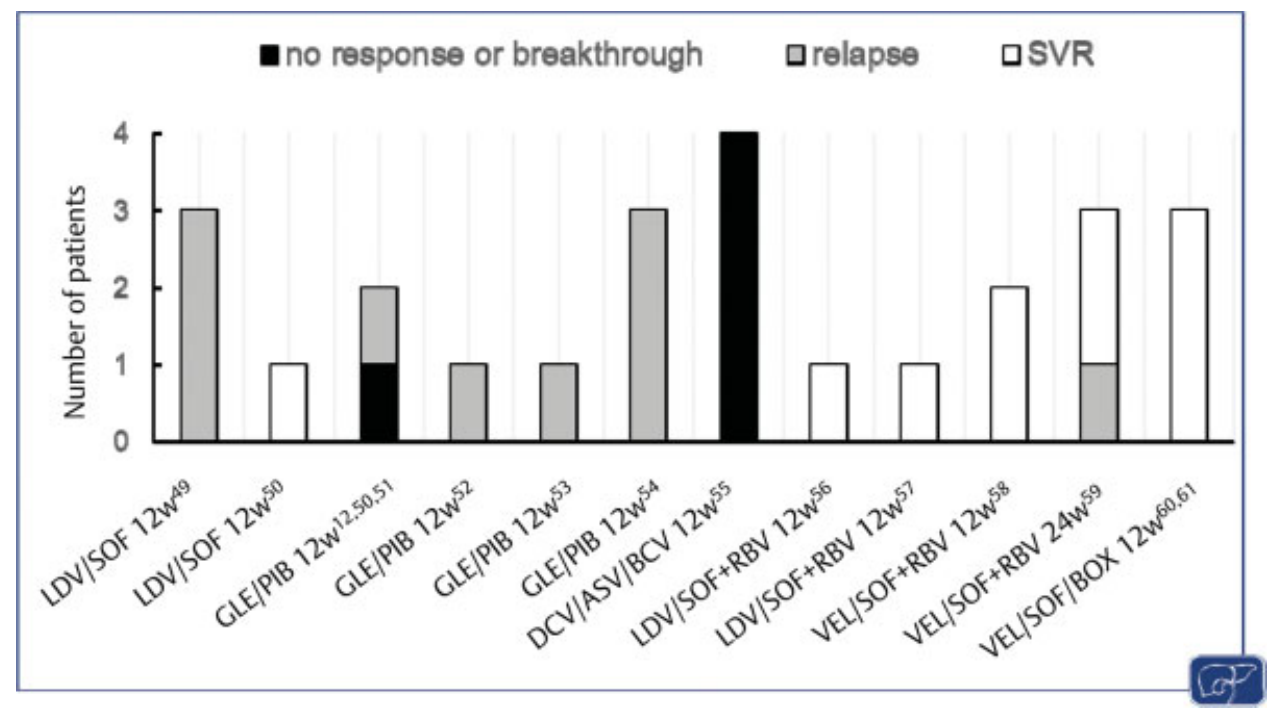

Fig. 2 Retreatment effect of patients infected with NS5A P32del hepatitis C virus (HCV). ASV, asunaprevir; BCV, beclabuvir; BOX, voxilaprevir; DCV, daclatasvir; GLE, glecaprevir; LDV, ledipasvir; PIB, pibrentasvir; RBV, ribavirin; SOF, sofosbuvir; VEL, velpatasvir. 
daclatasvir/asunaprevir/beclabuvir for 12 weeks; sofosbuvir/ ledipasvir + ribavirin for 12 weeks; sofosbuvir/velpatasvir + ribavirin for 24 weeks; and sofosbuvir/velpatasvir/voxilaprevir for 12 weeks. Takaguchi et al reported that a total of four patients with NS5A P32del HCV who had failed prior IFNfree DAA therapy were treated with daclatasvir/asunaprevir/ beclabuvir and that none of them achieved SVR despite having wild-type NS3 D168. ${ }^{56}$ Regarding daclatasvir/asunaprevir/ beclabuvir treatment, its efficiency against patients without NS5A P32del HCV who had failed prior IFN-free DAA therapy was also low, which was reported to be $33 \%$ (17/51) in realworld settings. ${ }^{56}$ Currently, daclatasvir/asunaprevir/beclabuvir are not recommended for any hepatitis $C$ patients, including patients with NS5A P32del HCV. Suda et $\mathrm{al}^{57}$ and Ikeda et $\mathrm{al}^{58}$ both reported one patient infected with NS5A P32del HCV who was retreated with sofosbuvir/ledipasvir + ribavirin for 12 weeks in two separate clinical trials. The two patients achieved SVR. In a Japanese phase 3 clinical trial for HCVinfected patients with prior DAA experience, five patients with NS5A P32del HCV were retreated with sofosbuvir/ velpatasvir + ribavirin. ${ }^{59}$ Among them, four patients achieved SVR, and only one patent developed virologic failure. In regard to sofosbuvir/velpatasvir/voxilaprevir, a total of three patients with NS5A P32del HCV were treated for 12 weeks in clinical trials (POLARIS-1, POLARIS-4), and all of them achieved SVR. ${ }^{60,61}$ The efficiency of sofosbuvir/velpatasvir/voxilaprevir, which includes both an NS3/4A inhibitor and an NS5B inhibitor but not an NS5A inhibitor, is expected to be high in patients with NS5A P32del HCV.

\section{Current Treatment Options for NS5A P32del HCV}

According to the current American Association for the Study of Liver Diseases guidelines, ${ }^{62}$ for NS5A Inhibitor DAA-experienced genotype $1 \mathrm{~b}$ patients with or without compensated cirrhosis, sofosbuvir/velpatasvir/voxilaprevir for 12 weeks is recommended. In the current European Association for the Study of the Liver guidelines, ${ }^{63}$ for DAA-experienced patients with or without compensated cirrhosis protease inhibitors and/ or NS5A inhibitor are recommended along with sofosbuvir/ velpatasvir/voxilaprevir for 12 weeks. For these patients, especially patients with predictors of a poor response (advanced liver disease, multiple courses of DAA-based treatment, and complex NS5A resistance-associated amino acid change profile), sofosbuvir + glecaprevir/pibrentasvir is recommended. To date, three patients with NS5A P32del HCV have been reported to be treated with sofosbuvir/velpatasvir/voxilaprevir, and all of them achieved SVR. The efficacy of sofosbuvir + glecaprevir/ pibrentasvir has not yet been reported in patients with NS5A P32del HCV. Although clinical evidence is weak, the $\mathrm{EC}_{50}$ of each drug against NS5A P32del HCV and experimental data using HCV-infected mice suggest that both treatments are expected to be effective against NS5A P32del HCV.

However, sofosbuvir/velpatasvir/voxilaprevir will not be developed in some Asian countries, including Japan, due to the race-specific toxicity of voxilaprevir. It is difficult to use sofosbuvir in combination with glecaprevir/pibrentasvir in some countries, including Japan, due to regulations related to the health insurance system. In these areas, other treatments containing more than one drug, except NS5A inhibitors such as sofosbuvir/velpatasvir + ribavirin, would be a suitable alternative choice. In regard to the sofosbuvir/velpatasvir + ribavirin treatment, 98\% (46/47) of NS5A DAA-experienced patients with HCV genotype 1b achieved SVR24 with sofosbuvir/velpatasvir + ribavirin retreatment for 24 weeks in a Japanese clinical trial ${ }^{59}$; thus, this regimen is the recommended treatment for DAA-experienced patients in the current guidelines of the Japan Society of Hepatology. Considering the high-level resistance of NS5A P32del HCV to velpatasvir, ${ }^{12,13,37,38}$ the effect of sofosbuvir/ velpatasvir + ribavirin treatment on patients with the NS5A P32del HCV is highly dependent on their sensitivity to sofosbuvir and ribavirin. The $\mathrm{EC}_{50}$ 's of sofosbuvir and ribavirin against NS5A P32del HCV are similar to those of wild-type HCV. ${ }^{37,38}$ NS5A P32del HCV is detected only in genotype $1 \mathrm{~b}$. The efficacy of sofosbuvir + ribavirin, which may not be as high as that of sofosbuvir/ledipasvir or sofosbuvir/velpatasvir, requires a longer treatment duration but is still relatively beneficial for naive patients when the HCV genotype is limited to genotype $1 \mathrm{~b}$. For example, a phase $3 \mathrm{~b}$ study in India demonstrated that sofosbuvir + ribavirin treatment for 24 weeks achieved $100 \%$ (23/23) SVR in treatment-naive genotype $1 \mathrm{~b}$ patients. ${ }^{64} \mathrm{~A}$ phase $3 \mathrm{~b}$ study in China demonstrated that sofosbuvir + ribavirin treatment for 24 weeks achieved 94\% SVR in NS5A inhibitor-unexperienced genotype 1b patients. ${ }^{65}$ Considering the efficacy of 24-week sofosbuvir + ribavirin treatment in genotype $1 \mathrm{~b}$ patients, velpatasvir/ sofosbuvir + ribavirin treatment for 24 weeks for patients with NS5A P32del HCV is expected to be effective and a suitable alternative choice when sofosbuvir/velpatasvir/voxilaprevir or sofosbuvir + glecaprevir/pibrentasvir cannot be used.

\section{Conclusion}

NS5A P32del was detected in 5 to $10 \%$ of genotype $1 \mathrm{~b}$ patients who experienced virologic failure with daclatasvir-containing therapies and sofosbuvir/ledipasvir treatment. Although most cases of NS5A P32del HCV are reported in Japan, some have been reported in clinical trials conducted in Europe ${ }^{53}$ and Western countries. ${ }^{60,61}$ These reports suggest that NS5A P32del HCV is relevant in countries other than Japan. NS5A P32del HCV clones are generated by the complete deletion of three bases, which encode NS5A P32 from the original clones. In regard to deep sequencing analysis, deletion of three consecutive bases is sometimes unmapped on the reference sequence and consequently cannot be detected. In the examination of HCV resistance-associated amino acid changes, confirming whether NS5A P32del HCV clones can be mapped appropriately on the used reference sequence is important. To date, NS5A P32del was not detected in naive patients. However, the possibility that a patient with NS5A P32del HCV infects someone cannot be excluded, especially among high-risk populations, such as persons who inject drugs. ${ }^{66,67}$ From this aspect, analysis of HCV resistance-associated amino acid changes in naive patients is also informative. A recommendation therapy 
against patients infected with NS5A P32del HCV remains unestablished. We should pay attention to the existence of NS5A P32del HCV, which is artificially generated by an NS5A inhibitor.

\section{Conflicts of Interest}

T.T. reports grants from the Japan Agency for Medical Research and Development, AMED, during the conduct of the study; grants and personal fees from Bristol-Myers Squibb, grants and personal fees from MSD K.K., grants and personal fees from AbbVie Inc, grants and personal fees from Gilead Sciences, grants from Chugai Pharmaceutical Co. Ltd, grants from Janssen Pharmaceutical K.K., outside the submitted work. H.H. reports grants from the Japan Agency for Medical Research and Development, AMED, during the conduct of the study; grants from MSD K.K., grants and personal fees from Bristol-Myers Squibb, outside the submitted work.

\section{References}

1 Foster GR, Afdhal N, Roberts SK, et al; ASTRAL-2 Investigators; ASTRAL-3 Investigators. Sofosbuvir and velpatasvir for HCV genotype 2 and 3 infection. N Engl J Med 2015;373(27):2608-2617

2 Mizokami M, Yokosuka O, Takehara T, et al. Ledipasvir and sofosbuvir fixed-dose combination with and without ribavirin for 12 weeks in treatment-naive and previously treated Japanese patients with genotype 1 hepatitis $C$ : an open-label, randomised, phase 3 trial. Lancet Infect Dis 2015;15(06):645-653

3 Alqahtani SA, Afdhal N, Zeuzem S, et al. Safety and tolerability of ledipasvir/sofosbuvir with and without ribavirin in patients with chronic hepatitis $C$ virus genotype 1 infection: analysis of phase III ION trials. Hepatology 2015;62(01):25-30

4 Reau N, Robertson MN, Feng HP, et al. Concomitant proton pump inhibitor use does not reduce the efficacy of elbasvir/grazoprevir: a pooled analysis of 1,322 patients with hepatitis $C$ infection. Hepatol Commun 2017;1(08):757-764

5 Wei L, Jia JD, Wang FS, et al; C-CORAL Investigators. Efficacy and safety of elbasvir/grazoprevir in participants with hepatitis $C$ virus genotype 1,4 , or 6 infection from the Asia-Pacific region and Russia: final results from the randomized C-CORAL study. J Gastroenterol Hepatol 2019;34(01):12-21

6 Puoti M, Foster GR, Wang S, et al. High SVR12 with 8-week and 12week glecaprevir/pibrentasvir therapy: an integrated analysis of HCV genotype 1-6 patients without cirrhosis. J Hepatol 2018;69 (02):293-300

7 Kumada H, Suzuki Y, Karino Y, et al. The combination of elbasvir and grazoprevir for the treatment of chronic HCV infection in Japanese patients: a randomized phase II/III study. J Gastroenterol 2017;52(04):520-533

8 Naganuma A, Chayama K, Notsumata K, et al. Integrated analysis of 8-week glecaprevir/pibrentasvir in Japanese and overseas patients without cirrhosis and with hepatitis $C$ virus genotype 1 or 2 infection. J Gastroenterol 2019;54(08):752-761

9 Carrasco I, Arias A, Benítez-Gutiérrez L, et al. Baseline NS5A resistance associated substitutions may impair DAA response in real-world hepatitis C patients. J Med Virol 2018;90(03): 532-536

10 Li DK, Chung RT. Overview of direct-acting antiviral drugs and drug resistance of hepatitis C virus. Methods Mol Biol 2019;1911:3-32

11 Dietz J, Susser S, Vermehren J, et al; European HCV Resistance Study Group. Patterns of resistance-associated substitutions in patients with chronic HCV infection following treatment with direct-acting antivirals. Gastroenterology 2018;154(04):976-988.e4
12 Gottwein JM, Pham LV, Mikkelsen LS, et al. Efficacy of NS5A inhibitors against hepatitis $C$ virus genotypes 1-7 and escape variants. Gastroenterology 2018;154(05):1435-1448

13 Krishnan P, Schnell G, Tripathi $R$, et al. Integrated resistance analysis of CERTAIN-1 and CERTAIN-2 studies in hepatitis C virus-infected patients receiving glecaprevir and pibrentasvir in Japan. Antimicrob Agents Chemother 2018;62(02):e02217-17

14 Gao M, Nettles RE, Belema M, et al. Chemical genetics strategy identifies an HCV NS5A inhibitor with a potent clinical effect. Nature 2010;465(7294):96-100

15 Fridell RA, Qiu D, Wang C, Valera L, Gao M. Resistance analysis of the hepatitis C virus NS5A inhibitor BMS-790052 in an in vitro replicon system. Antimicrob Agents Chemother 2010;54(09):3641-3650

16 Fridell RA, Wang C, Sun JH, et al. Genotypic and phenotypic analysis of variants resistant to hepatitis $C$ virus nonstructural protein $5 \mathrm{~A}$ replication complex inhibitor BMS-790052 in humans: in vitro and in vivo correlations. Hepatology 2011;54(06):1924-1935

17 Wang C, Huang H, Valera L, et al. Hepatitis C virus RNA elimination and development of resistance in replicon cells treated with BMS790052. Antimicrob Agents Chemother 2012;56(03):1350-1358

18 Nettles RE, Gao M, Bifano M, et al. Multiple ascending dose study of BMS-790052, a nonstructural protein 5A replication complex inhibitor, in patients infected with hepatitis $C$ virus genotype 1 . Hepatology 2011;54(06):1956-1965

19 Wang C, Sun JH, O'Boyle DR II, et al. Persistence of resistant variants in hepatitis $C$ virus-infected patients treated with the NS5A replication complex inhibitor daclatasvir. Antimicrob Agents Chemother 2013;57(05):2054-2065

20 McPhee F, Hernandez D, Zhou N, et al. Virological escape in HCV genotype-1-infected patients receiving daclatasvir plus ribavirin and peginterferon alfa-2a or alfa-2b. Antivir Ther 2014;19(05): 479-490

21 Uchida Y, Kouyama JI, Naiki K, et al. Development of rare resistance-associated variants that are extremely tolerant against NS5A inhibitors during daclatasvir/asunaprevir therapy by a two-hit mechanism. Hepatol Res 2016;46(12):1234-1246

22 Kai Y, Hikita H, Morishita N, et al. Baseline quasispecies selection and novel mutations contribute to emerging resistance-associated substitutions in hepatitis $C$ virus after direct-acting antiviral treatment. Sci Rep 2017;7:41660

23 Itakura J, Kurosaki M, Hasebe C, et al. Complex pattern of resistance-associated substitutions of hepatitis $\mathrm{C}$ virus after daclatasvir/asunaprevir treatment failure. PLoS One 2016;11 (10):e0165339

24 Kobayashi M, Akuta N, Suzuki F, et al. Treatment efficacy of all-oral combinations of direct-acting antivirals in chronic hepatitis $\mathrm{C}$ patients with NS5A-P32 deletion. Kanzo 2017;58(12):671-673

25 Iio E, Shintaro O, Shimada N, et al. Serial changes of NS5A P32deletion mutant in HCV genotype $1 \mathrm{~b}$ patients after Daclatasvir/Asunaprevir failure. Kanzo 2018;59(04):230-233

26 Teraoka Y, Uchida T, Imamura M, et al; Hiroshima Liver Study Group. Prevalence of NS5A resistance associated variants in NS5A inhibitor treatment failures and an effective treatment for NS5AP32 deleted hepatitis $C$ virus in humanized mice. Biochem Biophys Res Commun 2018;500(02):152-157

27 Doi A, Hikita H, Sakamori R, et al. Nonstructural protein 5A/P32 deletion after failure of ledipasvir/sofosbuvir in hepatitis $C$ virus genotype 1b infection. Hepatology 2018;68(01):380-383

28 Tellinghuisen TL, Marcotrigiano J, Gorbalenya AE, Rice CM. The NS5A protein of hepatitis C virus is a zinc metalloprotein. J Biol Chem 2004;279(47):48576-48587

29 Tellinghuisen TL, Marcotrigiano J, Rice CM. Structure of the zincbinding domain of an essential component of the hepatitis $C$ virus replicase. Nature 2005;435(7040):374-379

30 Penin F, Brass V, Appel N, et al. Structure and function of the membrane anchor domain of hepatitis $C$ virus nonstructural protein 5A. J Biol Chem 2004;279(39):40835-40843 
31 Ascher DB, Wielens J, Nero TL, Doughty L, Morton CJ, Parker MW. Potent hepatitis $\mathrm{C}$ inhibitors bind directly to NS5A and reduce its affinity for RNA. Sci Rep 2014;4:4765

32 Knops E, Sierra S, Kalaghatgi P, Heger E, Kaiser R, Kalinina OV. Epistatic interactions in NS5A of hepatitis $C$ virus suggest drug resistance mechanisms. Genes (Basel) 2018;9(07):E343

33 Nettles JH, Stanton RA, Broyde J, et al. Asymmetric binding to NS5A by daclatasvir (BMS-790052) and analogs suggests two novel modes of HCV inhibition. J Med Chem 2014;57(23):10031-10043

34 Yin C, Goonawardane N, Stewart H, Harris M. A role for domain I of the hepatitis $C$ virus NS5A protein in virus assembly. PLoS Pathog 2018;14(01):e1006834

35 Friborg J, Zhou N, Han Z, et al. In vitro assessment of re-treatment options for patients with hepatitis C Virus genotype $1 \mathrm{~b}$ infection resistant to daclatasvir plus asunaprevir. Infect Dis Ther 2014

36 Dvory-Sobol H, Han B, Lu J, et al. In vitro resistance profile of hepatitis C virus NS5A inhibitor velpatasvir in genotypes 1 to 6 . J Viral Hepat 2019;26(08):991-1001

37 Doi A, Hikita $\mathrm{H}$, Kai Y, et al. Combinations of two drugs among NS3/4A inhibitors, NS5B inhibitors and non-selective antiviral agents are effective for hepatitis $C$ virus with NS5A-P32 deletion in humanized-liver mice. J Gastroenterol 2019;54(05):449-458

38 Nitta S, Asahina Y, Kato T, et al. Impact of novel NS5A resistanceassociated substitutions of hepatitis $C$ virus detected in treatment-experienced patients. Sci Rep 2019;9(01):5722

39 Nitta S, Asahina Y, Matsuda M, et al. Effects of resistance-associated NS5A mutations in hepatitis $C$ virus on viral production and susceptibility to antiviral reagents. Sci Rep 2016;6:34652

40 Rhim JA, Sandgren EP, Degen JL, Palmiter RD, Brinster RL. Replacement of diseased mouse liver by hepatic cell transplantation. Science 1994;263(5150):1149-1152

41 Azuma H, Paulk N, Ranade A, et al. Robust expansion of human hepatocytes in Fah-/-/Rag2-/-/Il2rg-/- mice. Nat Biotechnol 2007; 25(08):903-910

42 Hasegawa M, Kawai K, Mitsui T, et al. The reconstituted 'humanized liver' in TK-NOG mice is mature and functional. Biochem Biophys Res Commun 2011;405(03):405-410

43 Washburn ML, Bility MT, Zhang L, et al. A humanized mouse model to study hepatitis $C$ virus infection, immune response, and liver disease. Gastroenterology 2011;140(04):1334-1344

44 Tsuge M, Fujimoto Y, Hiraga N, et al. Hepatitis C virus infection suppresses the interferon response in the liver of the human hepatocyte chimeric mouse. PLoS One 2011;6(08):e23856

45 Kai Y, Hikita H, Tatsumi T, et al. Emergence of hepatitis C virus NS5A L31V plus $\mathrm{Y} 93 \mathrm{H}$ variant upon treatment failure of daclatasvir and asunaprevir is relatively resistant to ledipasvir and NS5B polymerase nucleotide inhibitor GS-558093 in human hepatocyte chimeric mice. J Gastroenterol 2015;50(11):1145-1151

46 Kosaka K, Hiraga N, Imamura M, et al. A novel TK-NOG based humanized mouse model for the study of HBV and HCV infections. Biochem Biophys Res Commun 2013;441(01):230-235

47 Meuleman P, Libbrecht L, De Vos R, et al. Morphological and biochemical characterization of a human liver in a UPA-SCID mouse chimera. Hepatology 2005;41(04):847-856

48 Osawa M, Uchida T, Imamura M, et al. Efficacy of glecaprevir and pibrentasvir treatment for genotype $1 \mathrm{~b}$ hepatitis $\mathrm{C}$ virus drug resistance-associated variants in humanized mice. J Gen Virol 2019;100(07):1123-1131

49 Akuta N, Sezaki H, Suzuki F, et al. Ledipasvir plus sofosbuvir as salvage therapy for HCV genotype 1 failures to prior NS5A inhibitors regimens. J Med Virol 2017;89(07):1248-1254

50 Iio E, Shimada N, Takaguchi K, et al. Clinical evaluation of sofosbuvir/ledipasvir in patients with chronic hepatitis $\mathrm{C}$ geno- type 1 with and without prior daclatasvir/asunaprevir therapy. Hepatol Res 2017;47(12):1308-1316

51 Kumada H, Watanabe T, Suzuki F, et al. Efficacy and safety of glecaprevir/pibrentasvir in HCV-infected Japanese patients with prior DAA experience, severe renal impairment, or genotype 3 infection. J Gastroenterol 2018;53(04):566-575

52 Sano T, Akuta N, Suzuki F, et al. Role of NS5A-L31/Y93 double wildtype in failure of glecaprevir/pibrentasvir double therapy in two patients with a history of direct-acting antiviral agent failure: an ultra-deep sequencing analysis. Intern Med 2019;58(18):2657-2662

53 Poordad F, Pol S, Asatryan A, et al. Glecaprevir/Pibrentasvir in patients with hepatitis $C$ virus genotype 1 or 4 and past direct-acting antiviral treatment failure. Hepatology 2018;67(04):1253-1260

54 Osawa M, Imamura M, Teraoka Y, et al; Hiroshima Liver Study Group. Real-world efficacy of glecaprevir plus pibrentasvir for chronic hepatitis $C$ patient with previous direct-acting antiviral therapy failures. J Gastroenterol 2019;54(03):291-296

55 Uemura H, Uchida Y, Kouyama JI, et al. NS5A-P32 deletion as a factor involved in virologic failure in patients receiving glecaprevir and pibrentasvir. J Gastroenterol 2019;54(05):459-470

56 Takaguchi K, Toyoda H, Tsutsui A, et al. Real-world virological efficacy and safety of daclatasvir/asunaprevir/beclabuvir in patients with chronic hepatitis $C$ virus genotype 1 infection in Japan. J Gastroenterol 2019;54(08):742-751

57 Suda G, Ogawa K, Yamamoto Y, et al; NORTE Study Group. Retreatment with sofosbuvir, ledipasvir, and add-on ribavirin for patients who failed daclatasvir and asunaprevir combination therapy. J Gastroenterol 2017;52(10):1122-1129

58 Ikeda H, Watanabe T, Shimizu H, et al. Efficacy of ledipasvir/ sofosbuvir with or without ribavirin for 12 weeks in genotype $1 \mathrm{~b}$ $\mathrm{HCV}$ patients previously treated with a nonstructural protein $5 \mathrm{~A}$ inhibitor-containing regimen. Hepatol Res 2018;48(10):802-809

59 Izumi N, Takehara T, Chayama K, et al. Sofosbuvir-velpatasvir plus ribavirin in Japanese patients with genotype 1 or 2 hepatitis $C$ who failed direct-acting antivirals. Hepatol Int 2018;12(04):356-367

60 Bourlière M, Gordon SC, Flamm SL, et al; POLARIS-1 and POLARIS-4 Investigators. Sofosbuvir, velpatasvir, and voxilaprevir for previously treated HCV infection. N Engl J Med 2017;376(22):2134-2146

61 Sarrazin C, Cooper CL, Manns MP, et al. No impact of resistanceassociated substitutions on the efficacy of sofosbuvir, velpatasvir, and voxilaprevir for 12 weeks in HCV DAA-experienced patients. J Hepatol 2018;69(06):1221-1230

62 AASLD-IDSA HCV Guidance Panel. Hepatitis C guidance 2018 update: AASLD-IDSA recommendations for testing, managing, and treating hepatitis C virus infection. Clin Infect Dis 2018;67(10):1477-1492

63 European Association for the Study of the Liver. Electronic address: easloffice@easloffice.eu; European Association for the Study of the Liver. EASL Recommendations on Treatment of Hepatitis C 2018. J Hepatol 2018;69(02):461-511

64 Shah SR, Chowdhury A, Mehta R, et al. Sofosbuvir plus ribavirin in treatment-naïve patients with chronic hepatitis $C$ virus genotype 1 or 3 infection in India. J Viral Hepat 2017;24(05):371-379

65 Wei L, Xie Q, Hou JL, et al. Sofosbuvir plus ribavirin with or without peginterferon for the treatment of hepatitis $C$ virus: results from a phase 3b study in China. J Gastroenterol Hepatol 2018;33(06):1168-1176

66 Martinello M, Grebely J, Petoumenos K, et al. HCV reinfection incidence among individuals treated for recent infection. J Viral Hepat 2017;24(05):359-370

67 Rossi C, Butt ZA, Wong S, et al; BC Hepatitis Testers Cohort Team. Hepatitis $C$ virus reinfection after successful treatment with direct-acting antiviral therapy in a large population-based cohort. J Hepatol 2018;69(05):1007-1014 